\title{
Nível de atividade física para prevenção do excesso de gordura visceral em mulheres pós- menopáusicas: quanto é necessário?
}

\author{
Level of physical activity in the prevention of excess visceral \\ fat in postmenopausal women: how much is needed?
}

Cristiano Penas Seara Pitanga', Francisco José Gondim Pitanga ${ }^{2}$, Carmem Cristina Beck ${ }^{3}$, Ronaldo Eugénio Calçada Dias Gabriel ${ }^{4}$, Maria Helena Rodrigues Moreira ${ }^{5}$

${ }^{1}$ Centro Universitário Jorge Amado (Unijorge), Faculdade Regional da Bahia (Unirb), Universidade do Estado da Bahia, Educação a Distância (Uneb/ EaD), Salvador, BA, Brasil ${ }^{2}$ Departamento de Educação Física, Faculdade de Educação, Universidade Federal da Bahia (UFBA), Salvador, BA, Brasil ${ }^{3}$ Instituto Federal de Santa Catarina (IFSC), Campus Palhoça-Bilíngue, Ponte do Imaruim, SC, Brasil ${ }^{4}$ Departamento de Ciências do Desporto, Exercício e Saúde, Universidade de Trás-os-Montes e Alto Douro (UTAD). Centro de Investigação e Tecnologias AgroAmbientais e Biológicas, Portugal ${ }^{5}$ Departamento de Ciências do Desporto, Exercício e Saúde, UTAD. Centro de Investigação em Desporto, Saúde e Desenvolvimento Humano (CIDESD), Portugal

Correspondência para Cristiano Penas Seara Pitanga Coordenação de Educação Física, Campus Paralela, Unijorge Av. Luis Viana, 6775 41745-130 - Salvador, BA, Brasil cpspitanga@gmail.com

Recebido em 11/Dez/2011 Aceito em 21/Jul/2012

\begin{abstract}
RESUMO
Objetivo: Analisar a quantidade de atividade física (kcal/semana) necessária à prevenção do excesso de gordura visceral em mulheres pós-menopáusicas. Sujeitos e métodos: A amostra incluiu 239 mulheres com uma idade média de 57,2 anos. A área de gordura visceral (AGV) foi medida por bioimpedância octopolar e a atividade física, com a versão longa do IPAQ. Foram construídas curvas Receiver Operating Characteristic (ROC) e considerado um intervalo de confiança de 95\%. Resultados: As áreas sob a curva ROC para a atividade física moderada e a caminhada revelaram-se significativas. 0 dispêndio semanal de $1.601 \mathrm{kcal}$ na caminhada ou de 2.283 kcal de atividade física moderada foi identificado como bons valores de corte para prevenir o excesso de AGV. Conclusão: A prática da atividade física de intensidade leve a moderada, com um gasto calórico entre 1.601 e $2.283 \mathrm{kcal} /$ semana, previne o excesso de adiposidade central em mulheres pós-menopáusicas. Arq Bras Endocrinol Metab. 2012;56(6):358-63
\end{abstract}

Descritores

Atividade física; adiposidade central; gasto energético; pós-menopausa

\begin{abstract}
Objective: To analyze the level of physical activity (kcal/week) needed to prevent excess visceral fat in postmenopausal women. Subjects and methods: The sample included 239 women with a mean age of 57.2 years. Visceral fat area (VFA) was measured by octopolar bioelectrical impedance, and physical activity, by the long version IPAO. ROC Curves (Receiver Operating Characteristic) were drawn, and a 95\% confidence interval was used. Results: The areas under the ROC curve for moderate physical activity and walk proved to be significant. Weekly expenditure of 1,601 kcal in walking, or 2,283 kcal in moderate physical activity were identified as good cutoff values to prevent excess VFA. Conclusion: Mild to moderate physical activity, with a caloric expenditure between 1,601 and 2,283 kcal/week, prevents excess central adiposity in postmenopausal women. Arq Bras Endocrinol Metab. 2012;56(6):358-63
\end{abstract}

\section{Keywords}

Physical activity; central adiposity; energy expenditure; postmenopause

\section{INTRODUÇÃO}

Área de gordura visceral (AGV) é definida como a Aquantidade de gordura localizada na região intra- abdominal e vem sendo utilizada como um importante preditor de risco metabólico e cardiovascular $(1,2)$.

Os mecanismos pelos quais a gordura visceral aumentada pode influenciar a elevação do risco meta- 
bólico e cardiovascular podem ser parcialmente explicados pela excessiva produção de ácidos graxos livres na região abdominal visceral, os quais são distribuídos por meio da circulação portal expondo o fígado a elevadas concentrações de gordura, reduzindo a remoção hepática de insulina e resultando em hiperinsulinemia periférica, com o consequente aumento do risco de desenvolvimento de diabetes mellitus. A hiperinsulinemia pode também causar distúrbios nas concentrações dos lipídios plasmáticos, bem como hipertensão arterial (3).

Por sua vez, a atividade física está associada à diminuição dos níveis de gordura visceral $(4,5)$, pressão arterial (6), glicemia (7) e outros agravos metabólicos e cardiovasculares $(8,9)$. Estudos têm demonstrado que o estilo de vida ativo apresenta benefícios para a saúde de indivíduos de ambos os sexos $(10,11)$, porém a quantidade necessária para que esses resultados sejam alcançados permanece especulativa.

Além disso, especula-se que a atividade física reduz a gordura visceral em função do efeito lipolítico causado pela liberação das catecolaminas secretadas durante o movimento corporal, proporcionando, assim, altas taxas de lipólise na região abdominal (12). Com a redução da gordura visceral, consequentemente, os níveis de pressão arterial, glicemia e lipídios plasmáticos também são diminuídos.

A menopausa representa um marco que determina mudanças na vida da mulher, inclusive em seu papel social. Além disso, propicia sintomas desconfortáveis e aumento na prevalência de doenças. Entre elas, destaca-se a doença cardiovascular, hipertensão, alterações lipídicas e diabetes, que, de fato, são mais frequentes quando comparadas à fase pré-menopausa (13).

Recentes investigações têm demonstrado que a quantidade de 185 a 285 minutos por semana de atividade física moderada provoca benefícios na prevenção de diabetes em adultos de ambos os sexos. Além disso, esses mesmos estudos observaram que a caminhada isoladamente parece não trazer benefícios significativos para a prevenção do diabetes, principalmente em homens $(14,15)$.

Este estudo apresenta relevância porque o conhecimento da quantidade de atividade física mais adequada para prevenção e tratamento da gordura visceral poderá ser utilizado em ações de saúde que visem tanto à prevenção quanto ao tratamento de agravos metabólicos e cardiovasculares em mulheres pós-menopáusicas. Diante disso, o objetivo deste estudo é investigar a quantidade de atividade física necessária para a prevenção do excesso de gordura visceral em mulheres pós-menopáusicas.

\section{SUJEITOS E MÉTODOS}

\section{Desenho e local do estudo}

Este estudo de corte transversal insere-se no Projeto Menopausa em Forma, aprovado pela Fundação Portuguesa para a Ciência e Tecnologia e desenhado para examinar a influência do exercício físico no risco cardiovascular e na aptidão física e funcional de mulheres pós-menopáusicas (16).

As mulheres que integraram o programa foram selecionadas de um grupo de voluntárias que responderam a anúncios de jornal, panfletos distribuídos na comunidade, reportagens na televisão e rádio ou que foram encaminhadas por um médico. O recrutamento da amostra foi realizado no conselho de Vila Real (Portugal), entre os meses de novembro de 2005 e março de 2006 e os critérios de inclusão foram observados por meio da avaliação da história clínica e reprodutiva, tendo sido obtido de todas as participantes o consentimento informado assinado.

Todas as avaliações foram conduzidas por dois avaliadores técnica e cientificamente treinados para o efeito e supervisionados pelos investigadores. O estudo atendeu aos procedimentos da Declaração de Helsinqui (17) e foi aprovado pelo Conselho de Ética da Universidade de Trás-os-Montes e Alto Douro em Portugal e pela Fundação de Ciência e Tecnologia, também de Portugal, referência POCI/DES/59049/2004.

\section{Amostra}

A amostra foi constituída por 239 mulheres pós-menopáusicas com uma idade média de 57,4 \pm 6,6 anos, respeitando os seguintes critérios de inclusão: (a) ausência de menopausa precoce, (b) inexistência de significativa doença renal, hepática ou hematológica; (c) não existência de sintomas de angina de peito ou de infarte do miocárdio nos últimos três meses; (d) não utilização de medicação anti-hipertensora ou antiarrítmica; (e) ausência de uma hipertensão descontrolada (pressão arterial sistólica $\geq 200 \mathrm{mmHg}$ e/ou pressão arterial diastólica $\geq 105 \mathrm{mmHg}$ ) e; (f) inexistência de condições musculoesqueléticas passíveis de condicionarem a prática de exercício ou serem exacerbadas por este.

\section{Variáveis de estudo}

O estudo incluiu a análise da área de gordura visceral (AGV) e do nível de atividade física em gasto calórico ( $\mathrm{kcal} / \mathrm{semana})$, considerando diferentes intensidades 
(caminhada, moderada e vigorosa) e o total da atividade física (caminhada + moderada + vigorosa). Para efeitos de caracterização da amostra, são também apresentados os valores do peso e da altura.

\section{Instrumentação/Procedimentos}

A altura foi avaliada com o estadiômetro SECA 220 (Seca Corporation, Hamburg Germany) de acordo com os procedimentos definidos por Heyward e Wagner (18) e considerando um limite de tolerância de $2 \mathrm{~mm}$ (19). O peso e a AGV foram medidos com a bioimpedância octopolar InBody 720 (20) cumprindo os procedimentos especificados na literatura. Essa bioimpedância utiliza oito eletrodos, dois em contato com a palma (E1, E3) e o polegar (E2, E4) de cada mão e dois em contato com a parte anterior (E5, E7) e posterior (E6, E8) da planta de cada pé. Cinco impedâncias segmentares (braço direito, braço esquerdo, perna direita, perna esquerda e tronco) são medidas a 1,5 , $50,250,500$ e $1000 \mathrm{KHz}$. Os pontos de contato do corpo com os eletrodos foram previamente limpos com um tecido eletrolítico recomendado pelo fabricante e foram observadas as seguintes normas de preparação: (1) estar em jejum, (2) não consumir álcool 48 antes do teste, (3) não realizar exercício de intensidade moderada à elevada nas 12 horas antes da avaliação, (4) não efetuar o exame perante a presença de um estado febril ou de desidratação, (5) não utilizar bijuterias metálicas ou implantes dentários com metal (quando passíveis de serem removidos), (6) não ingerir café, e (7) realizar a avaliação em fato de banho ou roupa interior.

Vários estudos documentam que o InBody 720 proporciona estimativas precisas da composição corporal total e segmentar $(21,22)$ e da AGV $(23)$. As avaliações foram realizadas em jejum pelo mesmo técnico, e os dados foram eletronicamente importados para o Excel, usando o software Lookin'Body 3.0 (Biospace, Seoul, Coreia). Os erros técnicos do peso, da altura e da AGV foram obtidos, com base em medições em duplicado em 10 mulheres pós-menopáusicas, por meio da fórmula $\mathrm{ET}=$ $\left(\Sigma d^{2} / 2 n\right)^{0,5}$ ( $d$ é a diferença entre as duas avaliações e $n, \mathrm{o}$ número de elementos da amostra). Os valores calculados foram, respectivamente, $0,06 \mathrm{~kg}, 0,09 \mathrm{~m}$ e $0,97 \mathrm{~cm}^{2}$.

A atividade física foi avaliada por meio da versão longa do International Physical Activity Questionnaire (IPAQ), reunindo questões relacionadas com a frequência e duração das atividades físicas realizadas por mais de dez minutos contínuos durante a última semana e abrangendo quatro domínios de atividade física (trabalho, meio de transporte, doméstico e lazer). A validade e a confiabilidade deste questionário estão documentadas na literatura (24).

Cada um dos domínios de AF foi indicado em minutos/semana por meio da multiplicação da frequência semanal pela duração de cada uma das atividades realizadas. Para o cálculo do gasto energético, foi multiplicado o valor do dispêndio de energia de acordo com a atividade realizada, considerando-se sua duração e frequência semanal (tempo em minutos/semana). A conversão dos dados obtidos pelo IPAQ em medida de equivalente metabólico (METs) foi realizada de acordo com a proposta de Heymsfield e cols. (25), sendo considerados os seguintes valores para cada domínio: trabalho (3,3 METs, caminhada; 4,0 METs, atividade moderada; 8,0 METs, atividade vigorosa), transporte (3,3 METs, caminhada; 6,0 METs, deslocação com bicicleta), atividades domésticas (4,0 METs, atividade moderada no jardim ou quintal; 3,0 METs, atividade moderada dentro de casa; 5,5 METs, atividade vigorosa no jardim ou no quintal) e tempo livre (3,3 METs, caminhada; 4,0 METs, atividade moderada; 8,0 METs, atividade vigorosa).

O produto do valor de METs da atividade física pela sua duração e frequência resultou no gasto calórico em METs - minutos/semana, e sua conversão em kcal/semana foi obtida pela fórmula: METs-minutos/semana $\times$ [Peso $(\mathrm{kg}) / 60 \mathrm{~kg}$ ]. O questionário foi aplicado e analisado pelo mesmo técnico e obedeceu às orientações de processamento e de análise dos dados do IPAQ, disponíveis no site www.ipaq.ki.se.

\section{Análise estatística}

O poder preditivo e os pontos de corte da atividade física para a prevenção do excesso de gordura visceral foram identificados por meio das curvas Receiver Operating Characteristic (ROC), frequentemente utilizadas para a determinação de pontos de corte em testes diagnósticos ou de triagem (26).

Inicialmente foi identificada a área total sob a curva ROC entre atividade física e a prevenção do excesso de gordura visceral. Quanto maior a área sob a curva ROC, maior o poder discriminatório. Foi utilizado intervalo de confiança (IC) a 95\%. O cálculo do IC a 95\% determina se a capacidade preditiva não é devido ao acaso, não devendo seu limite ser inferior a 0,50 (27). Na sequência, foram calculadas a sensibilidade e 
a especificidade, além dos pontos de corte da atividade física para prevenção do excesso de gordura visceral. Valores identificados por intermédio da curva ROC constituem-se em pontos de corte que deverão promover um mais adequado equilíbrio entre sensibilidade e especificidade. Os dados foram analisados utilizando-se o programa estatístico STATA (versão 7.0, Stata Corp., College Station, Estados Unidos).

\section{RESULTADOS}

A análise descritiva da amostra é ilustrada na tabela 1 e destaca-se evidenciando seus elementos uma idade média de 57,2 anos $( \pm 6,6)$ e valores de AGV entre 45,8 e $108,7 \mathrm{~cm}^{2}$. O gasto energético semanal em atividade física habitual foi de $5411,5( \pm 6937,2) \mathrm{kcal} / \mathrm{semana}$, sendo obtido, sobretudo, com a prática de atividade de intensidade moderada $(3134,8 \mathrm{kcal} /$ semana $)$.

Tabela 1. Análise descritiva da amostra $(n=239)$

\begin{tabular}{lcc}
\hline Variáveis & Média \pm DP & Amplitude \\
\hline Idade (anos) & $57,2 \pm 6,6$ & $40,6-79,6$ \\
Peso $(\mathrm{kg})$ & $69,1 \pm 11,3$ & $45,8-108,7$ \\
Altura $(\mathrm{m})$ & $1,55 \pm 5,2$ & $1,42-1,70$ \\
Área de gordura visceral (cm²) & $134,5 \pm 26,9$ & $42,2-206,1$ \\
Atividade física habitual (kcal/semana) & & \\
\multicolumn{1}{l}{ Caminhada } & $1724,4 \pm 2549,9$ & $0-15178,1$ \\
\multicolumn{1}{c}{ Moderada } & $3134,8 \pm 4213,8$ & $0-25765,5$ \\
Total & $552,2 \pm 2467,1$ & $0-22304,8$ \\
\hline
\end{tabular}

DP: desvio-padrão.

A tabela 2 apresenta as áreas sob a curva ROC entre o nível de atividade física ( $\mathrm{kcal} / \mathrm{semana}$ ) e a prevenção do excesso de gordura visceral. As áreas sob a curva ROC para a atividade física total, moderada e caminhada revelaram significado estatístico $(\mathrm{p} \leq 0,05)$. A AF vigorosa não foi boa preditora para ausência do excesso de gordura visceral.

Tabela 2. Áreas sob a curva ROC e IC95\% entre a quantidade de atividade física (kcal/semana) como preditor da ausência de gordura visceral

\begin{tabular}{lc}
\hline Atividade física & AUC \\
\hline Caminhada & $0,73(0,63-0,83)^{\star}$ \\
Moderada & $0,63(0,53-0,73)^{\star}$ \\
Vigorosa & $0,56(0,47-0,66)$ \\
Total & $0,70(0,60-0,80)^{\star}$ \\
\hline
\end{tabular}

AUC: área sob a curva ROC apresentando poder discriminatório para a ausência de gordura visceral (Li-IC $\geq 0,50) ;{ }^{*} p \leq 0,05$.
Na tabela 3, podem ser observados os níveis de atividade física ( $\mathrm{kcal} / \mathrm{semana}$ ) mais adequados para a prevenção do excesso de gordura visceral nas mulheres pós-menopáusicas.

Tabela 3. Pontos de corte, sensibilidade e especificidade da quantidade de AF (kcal/semana) como preditor da ausência de gordura visceral

\begin{tabular}{lccc}
\hline Atividade física & $\begin{array}{c}\text { Ponto de corte } \\
\text { (kcal/semana) }\end{array}$ & $\begin{array}{c}\text { Sensibilidade } \\
\text { (\%) }\end{array}$ & $\begin{array}{c}\text { Especificidade } \\
\text { (\%) }\end{array}$ \\
\hline Caminhada & 1.601 & 8,2 & 68,8 \\
Moderada & 2.283 & 68,2 & 60,0 \\
Vigorosa & NR & - & - \\
Total & NR & - & - \\
\hline
\end{tabular}

NR: indicador não recomendável para a predição da ausência de gordura visceral (pontos de corte não apresentam boa sensibilidade e especificidade).

Os dados revelam que $1.601 \mathrm{kcal} / \mathrm{semana}$ de caminhada e/ou $2.283 \mathrm{kcal} / \mathrm{semana}$ de atividade física moderada constituem os melhores pontos de corte para predizer a prevenção para ausência do excesso de gordura visceral.

\section{DISCUSSÃO}

O estudo demonstra o poder discriminatório do nível de atividade física ( $\mathrm{kcal} / \mathrm{semana})$ na prevenção do excesso de gordura visceral em mulheres pós-menopáusicas, identificando valores de corte do gasto calórico semanal considerando adequado equilíbrio entre a sensibilidade e a especificidade. Os resultados revelam que um dispêndio energético semanal entre 1.601 e $2.283 \mathrm{kcal}$, articulado à prática de atividade física de intensidade leve a moderada, previne o excesso de adiposidade intra-abdominal em mulheres na pós-menopausa, não sendo reconhecida uma boa capacidade preditiva da atividade física de intensidade vigorosa. A relevância desses resultados na promoção da atividade física e da saúde das mulheres pós-menopáusicas é justificada por vários fatores. Em primeiro lugar, é reconhecido o efeito da depleção estrogênica no aumento da massa gorda e na aquisição de uma topografia corporal do tipo androide (28) concorrendo o incremento dos níveis de adiposidade, particularmente central, para a ampliação do risco cardiovascular na mulher (29) e para o comprometimento da sua condição muscular (30) e óssea (31). O excesso de adiposidade visceral está também associado a um maior risco de câncer da mama em mulheres nessa fase do climatério (32), sendo documentada, em estudo recente (33), a presença de me- 
nores níveis de aptidão aeróbia quando o excesso de adiposidade central coabita com a sarcopenia, independentemente da idade e do tempo de menopausa.

Revelando uma importante influência genética, o aumento da adiposidade visceral pode ser atenuado em mulheres pós-menopáusicas em cerca de 30\%, com a adoção de uma prática regular de atividade física (34). De acordo com o United States Department of Health and Human Services, um dispêndio energético entre 13 a $26 \mathrm{kcal} . \mathrm{kg}$.hora, sem restrição calórica, resulta na diminuição da adiposidade intra-abdominal (35).

A presente investigação documenta que a caminhada, utilizada em contexto de deslocamento, em atividades ocupacionais e domésticas e/ou no tempo de lazer, gera uma menor adiposidade visceral quando o gasto energético associado à sua prática ultrapassa $1.601 \mathrm{kcal}$ por semana. Sendo uma atividade física acessível à maior parte das mulheres pós-menopáusicas, não requerendo equipamento sofisticado ou material técnico específico e constituindo uma das suas atividades físicas de eleição, a caminhada constitui, assim, uma proposta atraente de promoção de um estilo de vida mais ativo nessa população.

O estudo revelou também que um dispêndio energético associado à prática de atividade moderada e superior a $2.283 \mathrm{kcal} / \mathrm{semana}$ foi um bom preditor no controle dos níveis de adiposidade visceral na pós-menopausa, o mesmo não acontecendo para a atividade física vigorosa. Reconhecendo que a mulher nessa fase do climatério tem preferência pela prática de atividades físicas moderadas em oposição às vigorosas (36), conferindo-lhes às primeiras maior bem-estar psicológico, sentimento de energia, autoeficácia e reforço positivo, particularmente na presença de um excesso de peso e/ ou estilo de vida sedentário (37), o cumprimento desses níveis de intensidade e de dispêndio energético revela-se importante na atenuação nos ganhos de adiposidade central e da perda das componentes da massa isenta de gordura, conferindo à mulher bem-estar psicológico e contribuindo para o aumento da sua adesão ao exercício. Pesquisas relatam (38) e com base nas orientações de atividade física definidas pelo American College of Sports Medicine (39), a mulher pós-menopáusica deverá cumprir pelo menos 150 minutos de atividade moderada por semana, por períodos com pelo menos $10 \mathrm{mi}-$ nutos de duração, período mínimo reconhecido como suficiente para melhorar a aptidão aeróbia e alguns fatores de risco para doença cardíaca e diabetes melito do tipo 2. A caminhada e as atividades físicas moderadas poderão ser desenvolvidas em distintos contextos (recreacional, transporte, ocupacional, doméstico etc.) e deverão estar orientadas para a melhoria da aptidão física e para a redução do risco de queda, de depressão, do declínio cognitivo e da manifestação de condições clínicas não infecciosas de longa duração. A elevação dos níveis de atividade moderada para 300 minutos por semana traz benefícios adicionais para a saúde da mulher, prevenindo o aumento ponderal e reduzindo o cancro da mama. O abatimento deste último poderá ser mais pronunciado se a mulher conjugar a prática de atividade física de intensidade moderada a vigorosa com outros fatores associados ao seu estilo de vida, como a restrição de hábitos tabágicos e alcoólicos e a redução do consumo de gorduras.

O impacto da atividade moderada nos níveis de adiposidade central depende da regularidade da sua prática, sendo aventados por alguns autores períodos de intervenção de pelo menos um ano, com implicações favoráveis na qualidade de vida da mulher pós-menopáusica.

As limitações deste estudo incluem o número de elementos da amostra e as metodologias utilizadas na apreciação da AGV e dos níveis de atividade física. Apesar de a validade do InBody 720 estar documentada na literatura, não constitui um método de referência para a apreciação da adiposidade intra-abdominal. No que se reporta à quantificação da atividade física habitual, a medição do gasto energético pelo registro das acelerações ao longo do tempo, recorrendo ao uso de sensores eletrônicos de movimento, proporciona estimativas mais rigorosas dos níveis de atividade física, comparativamente aos questionários.

Em conclusão, os valores de corte identificados na presente investigação revelam-se úteis na monitorização e na prescrição da atividade física em mulheres pós-menopáusicas, objetivando a obtenção de níveis de adiposidade central mais recomendáveis à saúde na pós-menopausa. É sugerida a prática de níveis de intensidade leve a moderada, satisfazendo um dispêndio calórico semanal entre 1.601 e $2.283 \mathrm{kcal}$.

Declaração: os autores declaram não haver conflitos de interesse científico neste estudo.

\section{REFERÊNCIAS}

1. Kotronen A, Yki-Järvinen $H$, Sevastianova $K$, Bergholm R, Hakkarainen $\mathrm{A}$, Pietiläinen $\mathrm{KH}$, et al. Comparison of the relative contributions of intra-abdominal and liver fat to components of the metabolic syndrome. Obesity. 2011;19(1):23-8. 
2. Seo JA, Kim BG, Cho H, Kim HS, Park J, Baik SH, et al. The cutoff values of visceral fat area and waist circumference for identifying subjects at risk for metabolic syndrome in elderly Korean: Ansan Geriatric (AGE) cohort study. BMC Public Health. 2009;2(9):443-9.

3. Dugan SA, Everson-Rose SA, Karavolos K, Avery EF, Wesley $\mathrm{DE}$, Powell LH. Physical activity and reduced intra-abdominal fat in midlife African-American and white women. Obesity. 2010;18(6):1260-5.

4. Sasai $H$, Katayama $Y$, Nakata $Y$, Eto $M$, Tsujimoto $T$, Ohkubo $H$, et al.The effects of vigorous physical activity on intra-abdominal fat levels: a preliminary study of middle-aged Japanese men. Diabetes Res Clin Pract. 2010;88(1):34-41.

5. Wagmacker DS, Pitanga, FJG. Atividade física comparada a atividade física no tempo livre como fator de proteção para hipertensão arterial sistêmica. Rev Bras Ciên Mov. 2009;17(1).

6. Hu FB, Leitzmann MF, Stampfer MJ, Colditz GA, Willett WC, Rimm EB. Physical activity and television watching in relation to risk for type 2 diabetes mellitus in men. Arch Intern Med. 2001;161:1542-8.

7. Ellison RC, Zhang Y, Qureshi MM, Knox S, Arnett DK, Province MA. Lifestyle determinants of high-density lipoprotein cholesterol: the National Heart, Lung, and Blood Institute Family Heart Study. Am Heart J. 2004;147(3):529-35.

8. Pitanga FJG. Atividade física e lipoproteínas plasmáticas em adultos de ambos os sexos. Rev Bras Ciên Mov. 2001;9(4):25-31.

9. Kohl HM. Physical activity and cardiovascular disease: evidence for a dose response. Med Sci Sports Exerc. 2001;33:472-83.

10. Pitanga FJG, Lessa I. Associação entre atividade física no tempo livre e proteína C-reativa em adultos na cidade de Salvador, Brasil. Arq Bras Cardiol. 2009;92(4):302-6.

11. Björntorp P. Adipose tissue distribuition and function. Int $\mathrm{J}$ Obes (Lond). 1991;15:67-81.

12. Després JP, Pouliot MC, Moorjani S, Nadeau A, Tremblay A, Lupien PJ, et al. Loss of abdominal fat and metabolic response to exercise training in obese women. Am J Physiol. 1991;261(24) E159-67.

13. Wenger NK. Epidemiology of coronary heart disease in women. Rev Soc Cardiol Estado São Paulo. 1996;6:672-80.

14. Pitanga FJG, Almeida LAB, Freitas MM, Pitanga CPS, Beck CC. Padrões de atividade física em diferentes domínios e ausência de diabetes em adultos. Motricidade. 2010;6:5-17.

15. Pitanga FJG, Lessa I, Barbosa PJB, Barbosa SJO, Costa MC, Lopes AS. Atividade física na prevenção do diabetes em etnia negra: quanto é necessário?. Rev Ass Med Bras. 2010;56(6):697-704.

16. Moreira H. Menopausa em forma: programa de promoção de exercício e de saúde em mulheres pós-menopáusicas. In: Dantas E. Atividade física e envelhecimento saudável. Rio de Janeiro: Editora Shape; 2008. p. 181-201.

17. World Medical Association. World medical association declaration of Helsinki: ethical principles for medical research involving human subjects. 59.ed. Seoul: WMA General Assembly, 2008.

18. Heyward V, Wagner D. Applied body composition assessment. 2.ed. Human Kinetics: Champaign, 2004.

19. Sobral F. Curso de antropometria. Lisboa: ISEF-CDI, 1985.

20. Biospace. InBody 720 the precision body composition analyser: user's manual. Seoul: Biospace Co., Ltda.; 2004.

21. Ling A, Craena A, Slagboomb P, Gunne D, Stokkelc M, Westendorpa $R$, et al. Accuracy of direct segmental multi-frequency bioimpedance analysis in the assessment of total body and segmental body composition in middle-aged adult population. Clin Nutr. 2011;30(5):610-5.
22. Miyatake N, Tanaka A, Eguchi M, Miyachi M, Tabata I, Numata T. Reference data of multi frequencies bioelectric impedance method in Japanese. J Anti-Aging Med. 2009;6(3):10-4.

23. Ogawa $H$, Fujitani $K$, Tsujinaka T, Imanishi K, Shirakata $H$, Kantani A, et al. InBody 720 as a new method of evaluating visceral obesity. Hepatogastroenterology. 2011;58(105):42-4.

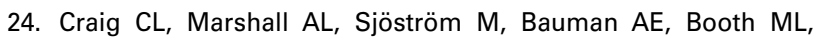
Ainsworth $\mathrm{BE}$, et al. International Physical Activity Questionnaire: 12-country reliability and validity. Med Sci Sports Exerc. 2003;35:1381-95.

25. Heymsfield SI, Lohman T, Wang Z, Going S. Human body composition. Ed. Champaingh, Human Kinetics, 2005.

26. Erdreich LS, Lee ET. Use of relative operating characteristics analysis in epidemiology: a method for dealing with subjective judgment. Am J Epidemiol. 1981;114(5):649-62.

27. Schisterman EF, Faraggi D, Reiser B, Trevisan M. Statistical inference for the area under the receiver operating characteristic curve in the presence of random measurement error. Am J Epidemiol. 2001;154(2):174-9.

28. Poehlman E, Toth M, Gardner A. Changes in energy balance and body composition at menopause: a controlled longitudinal study. Arch Intern Med. 1995;123(9):673-5.

29. Shuster L, Rhodes D, Goustout B, Rocca W. Premature menopause or early menopause: long-term health consequences. Menopause. 2010;65(2):161-6.

30. Schrager MA, Metter EJ, Simonsick E, Ble A, Bandinelli S, Lauretani $F$, et al. Sarcopenic obesity and inflammation in the InCHIANTI study. J Appl Physiol. 2007;102(3):919-25.

31. MacDonald P, Edman C, Hemsell D, Porter J, Siiteri P. Effect of obesity on conversion of plasma androstenedione to estrone in postmenopausal women with and without endometrial cancer. Am J Obstet Gynecol. 1978;130(4):448-55.

32. Lahmann PH, Lissner L, Gullberg B, Olsson H, Berglund G. A prospective study of adiposity and postmenopausal breast cancer risk: the Malmö Diet and Cancer Study. Int J Cancer. 2003;103(2):246-52.

33. Aragão $F$, Abrantes $C$, Gabriel $R$, Sousa M, Castelo-Branco $C$, Moreira $\mathrm{M}$. Effects of body composition and menopause characteristics on maximum oxygen uptake of postmenopausal women. Menopause. 2011;18(11):1191-7.

34. Demerath EW, Rogers NL, Reed D, Lee M, Choh AC, Siervogel $\mathrm{RM}$, et al. Significant associations of age, menopausal status and lifestyle factors with visceral adiposity in African-American and European-American women. Ann Hum Biol. 2011;38(3):247-56.

35. Friedenreich CM, Woolcott CG, McTiernan A, Terry T, Brant R, Ballard-Barbash $\mathrm{R}$, et al. Adiposity changes after a 1-year aerobic exercise intervention among postmenopausal women: a randomized controlled trial. Int J Obes. 2011;35(3):427-35.

36. Vallance JK, Murray TC, Johnson ST, Elavsky S. Understanding physical activity intentions and behavior in postmenopausal women: an application of the theory of planned behavior. Int $\mathrm{J}$ Behav Med. 2011;18(2):139-49.

37. Perez K, Garber C. Exercise prescritpion for the menopausal years: promoting and enhancing well-being. ACSMS Health Fit. 2011;15(3):8-14.

38. ACSM. ACSM's guidelines for exercise testing and prescription. 8.ed. Phildelphia:Wolters Kluwer - Lippincott Williams \& Wilkins, 2010.

39. Courneya KS, Tamburrini AL, Woolcott CG, McNeely ML, Karvinen $\mathrm{KH}$, Campbell KL, et al. The Alberta Physical Activity and Breast Cancer Prevention Trial: quality of life outcomes. Prev Med. 2011;52(1):26-32. 